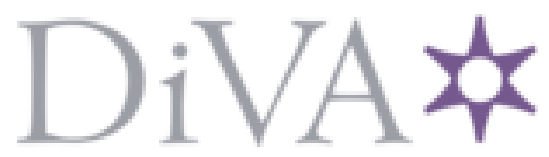

http://www.diva-portal.org

\title{
Postprint
}

This is the accepted version of a paper presented at IEEE SMC 2013, IEEE International Conference on Systems, Man, and Cybernetics, Manchester, UK, 13-16 October 2013.

Citation for the original published paper:

Maffei, A., Akillioglu, H., Flores, L. (2013)

Characterization of costs and strategies for automation in Evolvable Production Systems.

In: (ed.), IEEE conference proceedings

N.B. When citing this work, cite the original published paper.

(c) 2013 IEEE. Personal use of this material is permitted. Permission from IEEE must be obtained for all other users, including reprinting/ republishing this material for advertising or promotional purposes, creating new collective works for resale or redistribution to servers or lists, or reuse of any copyrighted components of this work in other works.

Permanent link to this version:

http://urn.kb.se/resolve?urn=urn:nbn:se:kth:diva-133436 


\section{Characterization of costs and strategies for automation in Evolvable Production Systems}

\author{
Antonio Maffei, Hakan Akillioglu \\ Dept. of Production Engineering \\ KTH The Royal Institute of Technology \\ Stockholm, Sweden \\ \{maffei, haaki\}@kth.se
}

\author{
Luis Flores \\ CTO, Engineering Director \\ Introsys - Global Control System Designers \\ Moita, Portugal \\ luis.flores@introsys.eu
}

\begin{abstract}
The rise of global competition and the demands for mass customization observed in recent years are the main shaping forces of the manufacturing domain. Current approaches to industrial production automation are not suitable to cope with the resulting increasing requirement in term of system agility and sustainability. While a quite large amount of innovative and sound technical solutions for automation address such an issue, it is not clear how the new generation of automatic production system will be economically connoted. This work proposes a first step towards an economical characterization of the Evolvable Paradigm: one among the most promising aforementioned innovative industrial automation technologies. A basic description of the state-of-the-art of the related Evolvable Assembly/Production System allows inferring a cost model able to account for such an installation. This, in turn, enable a quantitative description of how the focal innovative approach enables a more effective and rational use of industrial automation.
\end{abstract}

Keywords: Evolvable Production Systems, Cost Model, Automation Strategy.

\section{INTRODUCTION}

The exponential increase of information's availability, that follows the ITC recent development, has been impacting in many ways the consumer goods market. A generation of more and more aware and demanding customers is pushing toward the need for mass-customized products. In order to fulfill such exigency the boundaries of the competitive environment are nowadays set at global level. Firms with different political and socio-economic background are competing for the same slice of the market. This, in turn, poses heavy demands on manufacturing installations which must not only be more and more responsive to the changes imposed by volatile markets but also they should allow a sustainable growth for their stakeholders especially in western economies which does not benefit from relatively cheap labor cost and "manufacturerfriendly" regulation. Sustainability and agility are thus the goals for the next generation of production systems $[1,2]$.

Some of the current approaches to design and develop production systems seem to fail in meeting such envisaged requirements. In particular, traditional investment-intensive manufacturing facilities such as Automatic Assembly Systems
(AAS) are still conceived with a rigid, short-term oriented logic [3]. In detail, considering the two focal dimension of the issue:

- Scope: AAS are nowadays built around a set of foreseeable tasks. Extra task can be added only at the cost of expensive and time-consuming reengineering activities. This is true both for Dedicated Assembly System (DAS) and Flexible Assembly System (FAS) [4].

- Life-cycle. Once the AAS has finished his normal productive cycle, its reuse is limited only to some valuable part such as industrial robot or general purpose transport units. The effort related to every cycle of integration of such installations has only the reduced horizon of the particular production.

In conclusion, any AAS can be seen nowadays as a prototypic installation built by a set of System Integrators for a Company exploiting generic automation components and according to an "Engineer to Order" (ETO) logic.

Researchers and practitioners have been proposing since long time plenty of adequate solutions to tackle the challenges portrayed above and consequently define the next generation of industrial automatic production systems technologies. The drivers of such developments have been mainly: (1) the advancements in information technology and (2) the emerging of cheap and tiny powerful computational and memory hardware units.

This work focuses on one of the most promising approaches to achieve system agility and sustainability for automatic assembly installations: the Evolvable Paradigm. In view of this, and as a starting point for this work, the next paragraph §background, introduces and describes the related features of an Evolvable Production System (EPS).

Technical robustness is not the only enabling factor for such a novel approach. Conceiving and developing EPS needs sound economical and operational characterization in order to trigger and support the necessary investments [5]. This paper proposes a first step into this rather large and heterogeneous knowledge gap. First, an overall cost model for an EPS is presented and discussed. Such a construct, in turn, allows describing how EPS technology can be successfully used to 
implement a rationale and efficient stepwise process of production automation.

It is important to remark that this paper aims at building specific economical knowledge for a technology that has not yet reached the industrial automation market. Although, as mentioned before, several academic and professional contributions have been proposing the industrial use of agents through modular architectures of HW and SW (references), still, a significant mass of actual applications is lacking. As a consequence, the contributions presented hereby must be seen as the beginning of a supporting field of study that can be integrated and refined as the focal technology progresses to the market.

\section{BACKGROUND}

The first conceptual definition of the Evolvable Assembly System (EAS) Paradigm, or simply Evolvable Paradigm, is due to Onori [6]. Subsequent research efforts broadened the range of application of such principles from the assembly domain to the whole set of manufacturing unit processes: this was the dawn of the Evolvable Production System [7-9].

In recent years, the active EPS research community has extensively studied different aspects of such domain which, in turn has spread in different directions through a large amount of heterogeneous contributions: research requirements [10], design support tools and models $[11,12]$, business processes $[5,13,14]$, diagnostic $[15,16]$, system deployment and description [17, 18], system configuration [19], self-learning mechanisms [20], self-organization theory [21] and management of complexity [22] among the others. Most of the research efforts that shaped the EPS paradigm at the early stage have been carried out within the large collaborative European project EUPASS [23] while the more recent developments have been attained within the following European project IDEAS [24, 25].

Such latter project is also the main reference for the following classification (see Figure 1) of the elements of an EAS. An Evolvable Assembly System is built on an open and modular architecture. In order to support such proposition, the control approach is based on self-organization and selfconfiguration mechanisms attained at the cost of autonomous behaviors at modular level. The novelty of the approach if compared with other concurrent ones (such as Reconfigurable Manufacturing System [26, 27], Holonic Manufacturing System [28] or Bionic Manufacturing System [29, 30]) is mainly identifiable in the conceptualization of the production system as process specific entity rather than product specific one.

The EPS paradigm is based on the novel construct known as Mechatronic Agent, (MA). In general a MA is composed of three elements: an (1) Agent which is a piece of software able to foster a series of elementary behaviors. Those behaviors allow the agent to interact with other agents and exploits some (2) Skills. Skills are conceptual resources strictly related to one or more pieces of (3) Hardware that is the physical representation of the MA. Given the MA nature of hybrid hardware/software entity the controller which allocates the agent and the related skills is intended to be embedded with the rest of the HW.

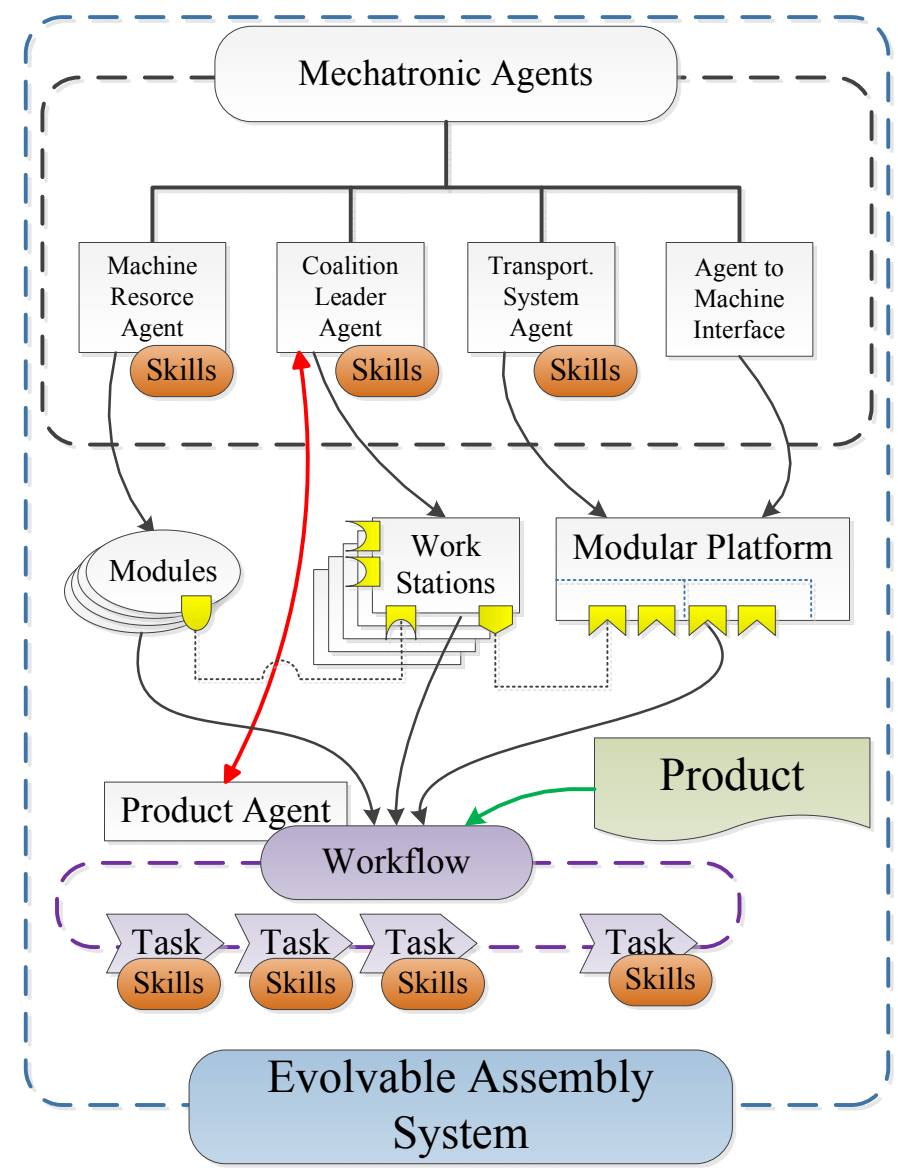

Figure 1 Overview of an EPS: elements and interconnections (adapted from [14])

Summarizing one can distinguish the following six innovative elements of an EAS:

1. The Multi-Agent System (MAS) [31]. It can be seen as the "Operative System" of the EPS. Practically is a modular piece of software which is logically and physically distributed across different hardware supports in the system, according to specific requirements [32]. It includes all the generic behaviors able to support the use of the skills.

2. Skill [33]. Skills are a powerful abstract construct introduced by the EPS research community with the aim of representing both the EPS process model (necessary to define the workflow), and of the EPS hardware (embodied into the platform, the workstation and the modules).

3. Workflow. This abstract construct represent all the information necessary to assemble a product as a sequence of skills. In view of this a workflow is nothing more than a sophisticated composition of skills and for this reason it is governed by a Coalition Leader Agent (CLA). The Workflow is 
related to a particular product and to the specific system meant to deliver it.

4. Modular Platform. This element is the physical embodiment of the internal logistic of the EPS and it is managed by a Transport System Agent (TSA). The platform is made of standard modules which, in turn, include: the interfaces to other platform modules, the standard slots for the workstations and the logistic between the different slots.

5. Workstation. This piece of hardware results from the aggregation of standard modules and it is devoted to execute a specific assembly task. From a process perspective it realizes an aggregation of skills (governed by a CLA).

6. Module. This construct represents a Machine Resource Agent (MRA). It supports an atomic skill.

In conclusion, it is possible to say that the innovation introduced by the EPS paradigm is represented though the depicted set of elements..

\section{COST MODEL FOR EVOLVABLE PRODUCTION SYSTEMS}

The depicted representation of the EPS paradigm and the related constructs allow at this point investigating the costs of buying and running such an installation. The usual classification of costs adopted for studying automatic installation is:

$$
\mathrm{C}_{\text {Total }}=\mathrm{C}_{\text {Fixed }}+\mathrm{C}_{\text {variable }}
$$

Fixed cost is that group of costs whose total will remain relatively constant throughout the range of operational activity. That is, fixed costs are more or less the same regardless of production rate, number of shifts or people. Such entry usually represents investments that are made in advance of the start of operation. Machinery, and therefore AAS, is usually accounted as fixed costs. Variable cost is that group of costs that vary in some relationship to the level of operational activity. Electricity, tools, gripper, fixtures are some examples in this category. People which work with supporting activities associated with the AAS can be seen theoretically as variable costs. For the limited purpose of this analysis it is possible to consider them in such way, but in reality union contracts and investment in training prevent company from easily lay off such resource.

In EPS such classification is not really useful: in principle all the hardware and software resources can be easily shifted from one production to another and they can be put in place in different moments of the lifecycle of the plant. Therefore the model presented herby will focus instead on the direct cost associated with such installation. In view of the EPS description used in this work, the related simplified cost can be represented by the following equation:

$$
\mathrm{C}_{\mathrm{EPS}}=\mathrm{C}_{\text {Platform }}+\sum_{\mathrm{i}=1}^{\mathrm{n}} \mathrm{C}_{\mathrm{WSi}}
$$

Where:

- $\mathrm{C}_{\text {platform }}=$ Cost of the platform. Included the cost required for setting the TSA and related skills.

- $\mathrm{C}_{\mathrm{WSi}}=$ Cost for the workstation $\mathrm{i}$

- $\mathrm{n}=$ Number of workstations in the system

It is possible to detail better the cost of the platform as:

$$
\mathrm{C}_{\text {Platform }}=\mathrm{K}+\sum_{\mathrm{l}=1}^{\mathrm{p}} \mathrm{f}_{\mathrm{Ul}} \mathrm{C}_{\mathrm{PUI}}
$$

Where:

- $\mathrm{K}=$ Cost for the integration of the platform. Included the cost required for setting the TSA and related Skills. This cost is not dependent from the number of units because the TSA and the related skills are scalable.

- $\quad \mathrm{C}_{\mathrm{PMI}}=$ Cost for the platform unit 1 .

- $\mathrm{p}=$ Number of platform unit in the system

- $\mathrm{f}_{\mathrm{Ul}}=$ Fraction of the total cost of the platform unit to be depreciated on the current production. Platforms are in fact not dependent on the particular production, but they serve different products.

The EPS modular architecture promises seamless reconfiguration and modules that can be seen as building blocks for several different applications. Theoretically this means that modules could be employed as flexible resources where they are needed within the company different line of products or even across different companies that could share the risk of owning them. This consideration allows at this point formulating the cost of a workstation accounting of the possibility of reusing the modules for other productions:

$$
\mathrm{C}_{\mathrm{WS}}=\mathrm{G}+\sum_{\mathrm{j}=1}^{\mathrm{m}} \mathrm{f}_{\mathrm{Mj}} \mathrm{C}_{\mathrm{Mj}}
$$

Where:

- $\mathrm{G}=$ Cost for the physical and logical integration of the workstation: including the cost required for setting the CLA and related Skills.

- $\mathrm{C}_{\mathrm{Mj}}=$ Cost of the Module $\mathrm{j}$. Included the cost required for setting the RMA and related Skills.

- $\mathrm{m}=$ Number of modules in the workstation.

- $\mathrm{f}_{\mathrm{Mj}}=$ Fraction of the module's overall cost to be depreciated on the current production.

The fixed cost for traditional automation, with some exceptions, must be put in place entirely before the production starts. This is mainly due to the integral architecture of such installations. Once again, thanks to the modular architecture and the irrelevant costs of integration and management of the system redundancies, EPS paradigm relaxes the constraints of buying the whole system capacity at the beginning. EPS Modules can be purchased according with the volume trend: this allow the companies to achieve a better balance in the cash flows. Fig. 2 shows, from a purely conceptual point of view, 
the impact of EPS on the financial exposition of a firm that uses an AAS.
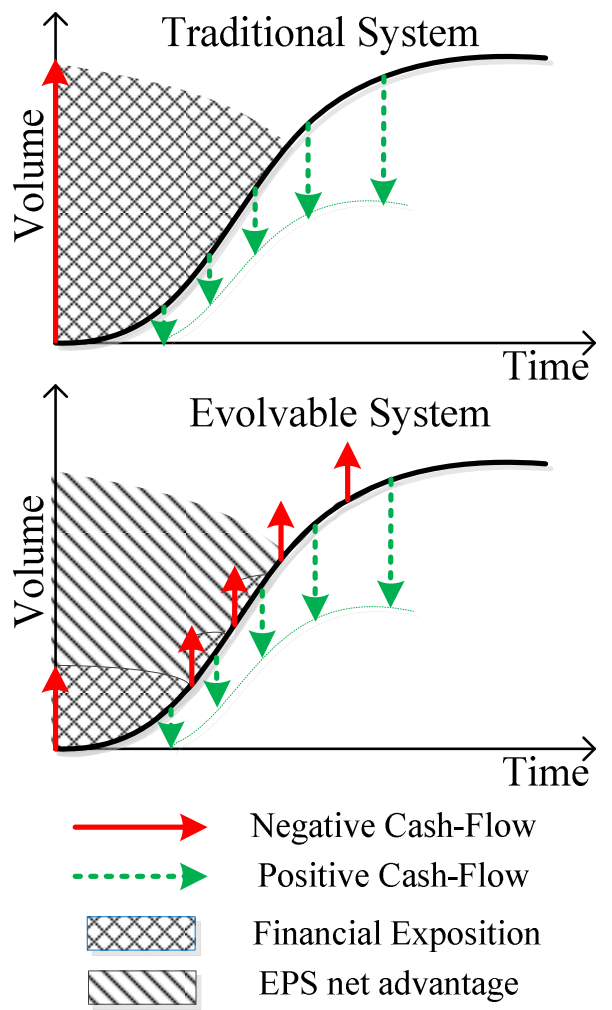

Figure 2 Cash Flow associated with automation: EPS vs. Traditional

This consideration allow to express the present value of the overall investment required for an EPS accounting on the fact that in EPS the negative cash flows related to automation occurs over the time in a discrete set of instants:

$$
\mathrm{I}_{\mathrm{EPS}}=\sum_{\mathrm{t}=\mathrm{t}_{1}}^{\mathrm{t}_{\mathrm{M}}} \mathrm{C}_{\mathrm{mt}}\left(1+\mathrm{r}_{\mathrm{t}}\right)^{-\mathrm{t}}
$$

Where:

- $\mathrm{C}_{\mathrm{mt}}=$ Cost for the modules purchased at the instant $\mathrm{t}$ as described in the following table. The modules can be both platform units and production modules.

Table 1 Purchasing time expressed as fraction of reference period starting at the instant 0

\begin{tabular}{|c|c|}
\hline Module & Purchasing time \\
\hline 1 & $\mathrm{t}_{1}$ \\
\hline 2 & $\mathrm{t}_{2}$ \\
\hline 3 & $\mathrm{t}_{3}$ \\
\hline$\ldots$ & $\ldots$ \\
\hline $\mathrm{M}^{\mathrm{a}}$ & $\mathrm{T}_{\mathrm{N}}$ \\
\hline \multicolumn{2}{|c|}{${ }^{\mathrm{a}} \mathrm{M}=$ total number of workstations }
\end{tabular}

- $\mathrm{r}_{\mathrm{t}}=\mathrm{t} * \mathrm{r}$.The rate of interest expressed as a fraction due at the instant $t$.
- $r=$ The rate of interest expressed as a fraction due each reference time period.

\section{EPS STEPWISE APPROACH TO AUTOMATION}

As seen in the previous paragraph, EPS modular architecture and the related seamless integration process offer to companies an unprecedented flexible approach to automation. On the one hand the full automatic solution can be bought at the beginning and scaled according to the volume just adding more platforms; on the other hand it is possible to buy automatic solutions only for a restricted set of process at the beginning, keeping the others manual, and then move towards full automation as the volume grows. Hybrids between these two scenarios are also possible. This paragraph investigates the second occurrence providing a simplified, yet valuable, tool to support a rationale stepwise approach to automation.

With reference to a generic product and assuming that:

1. The product is composed of $\mathrm{N}$ parts each one requiring a single task to be assembled;

2. Each task can be performed by a human operator with execution time equal to $\tau_{\mathrm{M}}$ expressed in the unit of time;

3. Human operator are considered fully flexible resource: therefore variable costs;

4. Each task can be performed by a specific automatic WS with execution time equal to $\tau_{\mathrm{A}}$ expressed in the unit of time;

5. The cost for the platform is only accounted when the first automatic workstation is deployed. Such cost is also proportional to the number of automatic workstations: in principle, in fact, an EPS platform is a flexible resource that can be employed according to the needs.

6. The variable costs associated with the automatic solution are following an exponential decay as the number of station increases. In particular the function of decreasing can be modeled with the following: $\quad v_{i}=\exp (-b i)$ where $b$ is a coefficient depending on the specific configuration of the cost.

7. The automatic WSs are introduced one at the time;

8. The cost of the money is not considered.

It is possible to define the following parametric expression for the overall costs:

$$
\left\{\begin{array}{rlr}
\mathrm{C}_{i}(V)= & \mathrm{C}_{0} \tau_{M} N V & \text { If } \mathrm{i}=0 \\
\mathrm{C}_{\mathrm{i}}(\mathrm{V})= & \mathrm{C}_{0} \tau_{M}(\mathrm{~N}-\mathrm{i}) \mathrm{V} / & \text { else } \\
& +\mathrm{i} \mathrm{I}_{\text {EPS }}+\tau_{A} v_{i} V
\end{array}\right.
$$

Where:

- $\mathrm{i}=$ The parameter in exam representing the number of automatic workstations in the system.

- $\mathrm{C}_{0}=$ Cost of the operator per unit of time.

- $\mathrm{V}=$ Product volumes.

- $\mathrm{I}_{\mathrm{EPS}}=\left(\mathrm{f}_{\mathrm{ws}} \hat{\mathrm{I}}_{\text {platform }}+\hat{\mathrm{I}}_{\mathrm{ws}}\right)$ 
Where:

- $\mathrm{f}_{\mathrm{ws}}=$ Fraction of the platform to be allocated on a workstation (assumed constant).

- $\quad \hat{\mathrm{I}}_{\text {platform }}=$ Fixed investment for expanding the platform each time a WS is added.

- $\quad \hat{\mathrm{I}}_{\mathrm{ws}}=$ Fixed investment for a Workstation.

The following Fig. 3 represents an indicative trend of this formulation for the production costs in function of the volume. Each different level of the parameter i "number of automatic station in the system" is represented by a line. The related parametric formulations for intercept and slope are also represented.

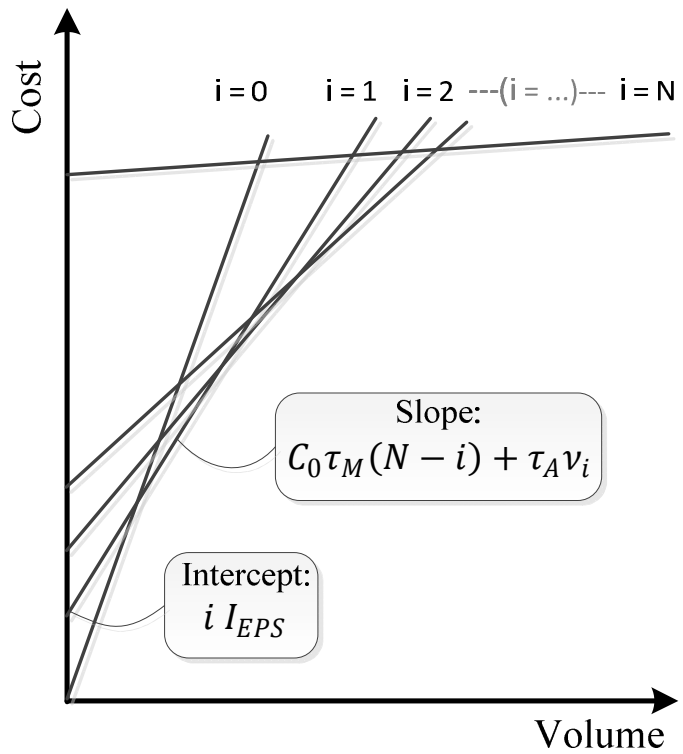

Figure 3 Graphical trend of the costs for a production system in function of the production volume and for different amount (i) of automatic stations in the system. (Adapted from [14])

The following function represents the minimum cost associated with producing a volume:

$$
\min _{\substack{0 \leq \mathrm{V} \leq \mathrm{V}_{\max } \\ 0 \leq \mathrm{i} \leq \mathrm{N}}} \mathrm{C}_{\mathrm{i}}(\mathrm{V})
$$

The locus that fulfills such condition is the polynomial chain represented with a thicker line in Fig. 4. The same Fig. 3 introduces also the parametric formulation of the coordinates of a generic interception point between $\mathrm{C}_{\mathrm{i}}(\mathrm{V})$ and $\mathrm{C}_{\mathrm{i}+1}(\mathrm{~V})$.

The value of $i$ in each segment of such polynomial chain represents the economically optimal number of automatic stations for the underlying production volumes. The value $\overline{\mathrm{V}} \mathrm{i}$ provides therefore an indication of the volume beyond which is economically convenient to shift from a manufacturing strategy based on $i$ automatic stations to one based on (i+1). Knowing the expected trend in the market demand for the product it is therefore possible to calculate the moment $T_{i}$ in which the $(i+1)^{\text {th }}$ automatic station should be deployed. A graphical example of this process is provided in Fig. 5 in the next page.

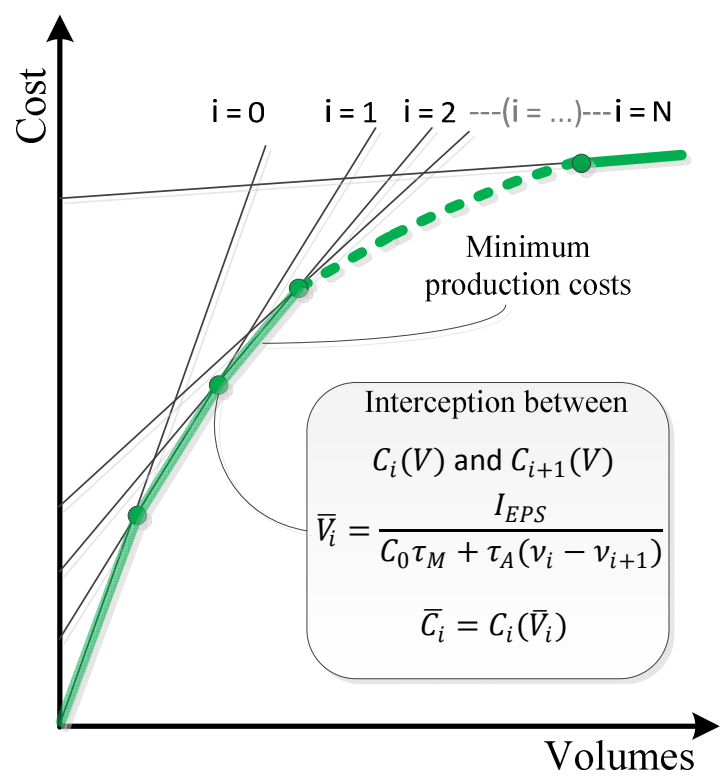

Figure 4 Polynomial chain representing the locus of minimum cost in function of the production volumes and for different amount (i) of automatic stations in the system. (Adapted from [14]).

Knowing the lead time $\mathrm{LT}_{\mathrm{i}+1}$ for the $(\mathrm{i}+1)^{\text {th }}$ automatic station it is the possible to express the ordering time for such station as following:

$$
\mathrm{OT}_{\mathrm{i}+1}=\mathrm{T}_{\mathrm{i}}-\mathrm{LT}_{\mathrm{i}+1}
$$

\section{DISCUSSION AND CONCLUSION}

The proposed cost model is able to capture and represent the innovative elements introduced by the EPS paradigm, such as the possibility to use the automatic general purpose elements throughout different productive cycles and the less demanding investment strategy required for such an installation. This latter yield of EPS technology is deeply investigated and characterized in the second contribution of this work, which suggests a quantitative analysis that supports a stepwise introduction of the focal automatic solution in a standard production scenario.

Both the cost model and the model for a stepwise approach to automation refers to a non-fully mature innovative technology, thus they have no use for current manufacturing facilities. Nevertheless they are useful tool to understand and describe the economical connotation of an EPS and thus can be proficiently used as complement for the technological input necessary for the final embodiment of such an innovative approach into actual industrial applications. In other words, they must be seen as supporting elements to boost awareness on the potential application of modular automatic solution featuring distributed control such as the EPS.

A future, concrete use of such contributions as actual reference model is subordinated to: the specific technological pattern that will lead EPS innovation to the market, and to the 
consequent integration and adaptation of the models themselves to the final mature stage of the technology.

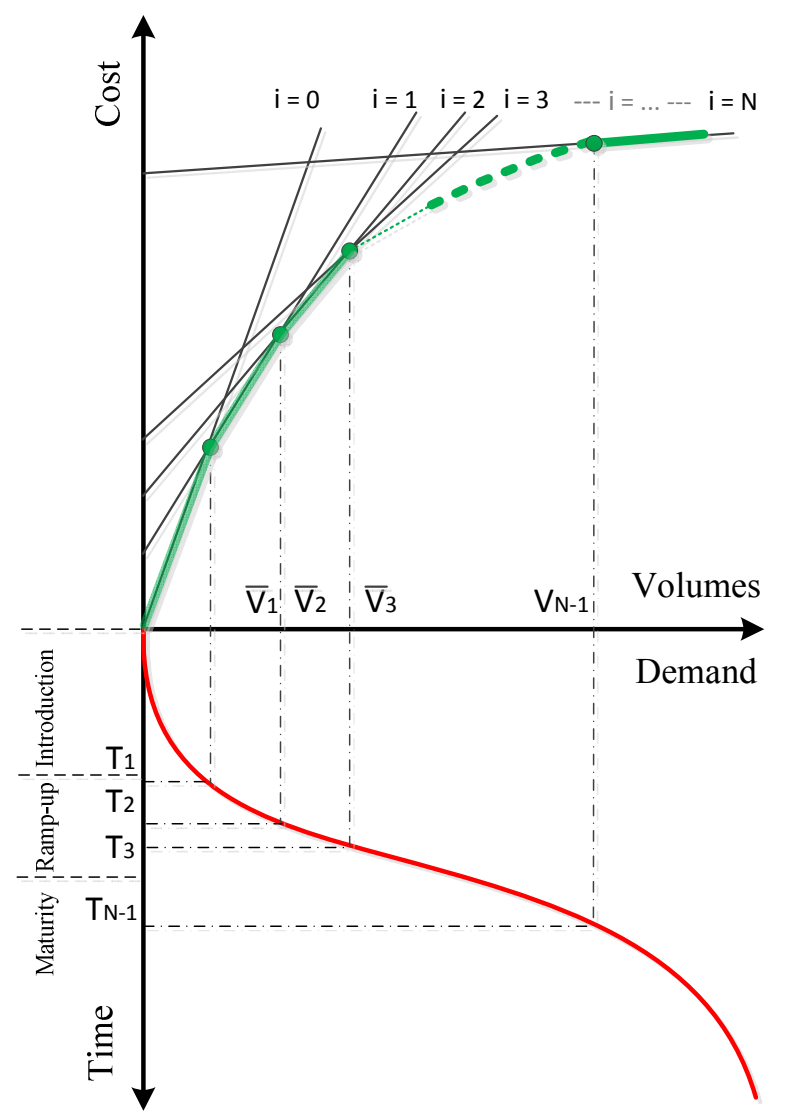

Figure 5 Graphical calculation of the economically optimal instants Ti of deployment for the (i+1) ${ }^{\text {th }}$ automatic station. (Adapted from [14])

\section{REFERENCES}

[1] EUPASS-ROADMAP, "EUPASS Adaptive Assembly Roadmap 2015 deliverable 1.5 f, Project Report-Public Document 1.5 f," NMP-2-CT-2004-5079782008.

[2] F. Jovane, E. Westkämper, and D. Williams, The ManuFuture Road: Towards competitive and sustainable high-adding-value manufacturing vol. 10: Springer, 2008.

[3] A. Maffei and M. Onori, "Evolvable Production Systems: Environment for New Business Models," Key Engineering Materials, vol. 467, pp. 1592-1597, 2011.

[4] A. Maffei and A. Hofmann, "From flexibility to true Evolvability: An introduction to the basic requirements," 2010, pp. 2658-2663.

[5] A. Maffei, P. Neves, and M. Onori, "Identification of the Value Proposition of an Evolvable Production System" presented at the 9th International Symposium on Mechatronics and its Applications (ISMA13), Amman, Jordan, 2013.

[6] M. Onori, "Evolvable assembly systems-a new paradigm?," in 33rd International Symposium on Robotics, Stockholm, Sweden, 2002, pp. 617-621.

[7] M. Onori and J. Barata, "Evolvable production systems: mechatronic production equipment with process-based distributed control," in 9th IFAC Symposium on Robot Control: SYROCO 2009, Gifu, Japan, 2009.

[8] J. Barata, M. Onori, and e. al., "Evolvable Production Systems: Enabling Research Domains.," presented at the International
Conference on Changeable, Agile, Reconfigurable and Virtual Production, Toronto, Canada, 2007.

[9] J. Barata, M. Onori, R. Frei, and P. Leitão, "Evolvable production systems in a RMS context: enabling concepts and technologies," 2007.

[10] J. Barata, P. Santana, and M. Onori, "Evolvable assembly systems: a development roadmap," 2006.

[11] N. Lohse, "Towards an ontology framework for the integrated design of modular assembly systems," University of Nottingham, 2006.

[12] N. Lohse, H. Hirani, and S. Ratchev, "Equipment ontology for modular reconfigurable assembly systems," International journal of flexible manufacturing systems, vol. 17, pp. 301-314, 2005.

[13] A. Maffei, "Evolvable production systems: foundations for new business models," Lic. Eng., Production Engineering, KTH ITM, Stockholm, 2010.

[14] A. Maffei, "Characterisation of the Business Models for Innovative, Non-Mature Production Automation Technology," $\mathrm{PhD}$, Production Engineering, Royal Institute of Technology, Stockholm, 2012.

[15] J. Barata, L. Ribeiro, and M. Onori, "Diagnosis on evolvable production systems," 2007, pp. 3221-3226.

[16] L. Ribeiro, "Diagnosis in evolvable production systems," $\mathrm{PhD}$, Electrical and computer engineering, FCT-UNL, Lisbon, 2012.

[17] A. Maffei and T. Rossi, "Development of an Ontology to support EAS: Electrolux Test Case," M.Sc., DIMNP, University of Pisa, Royal Institute of technology Stockholm, Pisa, Italy, 2007.

[18] N. Siltala, A. F. Hofmann, R. Tuokko, and G. Bretthauer, "Emplacement and blue print-An approach to handle and describe modules for evolvable assembly systems," in Proceedings of the IFAC/SYROCO 2009 Conference, Gifu, Japan, 2009, pp. 8691.

[19] P. Ferreira, "An agent-based self-configuration methodology for modular assembly systems," University of Nottingham, 2011.

P. Neves, "System Evaluation and Learning in Evolvable Production Systems," Licentiate, Production Engineering, The Royal Institute of Technology, Stockholm, 2012.

[21] R. Frei, G. Di Marzo Serugendo, and J. Barata, "Designing selforganization for evolvable assembly systems," in Self-Adaptive and Self-Organizing Systems, 2008. SASO'08. Second IEEE International Conference on, 2008, pp. 97-106.

[22] M. Bjelkemyr and A. Maffei, "Handling complexity in Evolvable Production Systems," 2010, pp. 2664-2668.

[23] EUPASS. (2004-2009). Evolvable Ultra-Precision Assembly Systems. Available: http://www.eupass-fp6.org/

[24] IDEAS, "Instantly Deployable Evolvable Assembly Systems," 2010-2013.

[25] M. Onori, N. Lohse, J. Barata, and C. Hanisch, "The IDEAS project: plug \& produce at shop-floor level," Assembly Automation, vol. 32, pp. 124-134, 2012.

[26] Z. Bi, S. Lang, W. Shen, and L. Wang, "Reconfigurable manufacturing systems: the state of the art," International Journal of Production Research, vol. 46, pp. 967-992, 2008.

[27] Y. Koren, U. Heisel, F. Jovane, T. Moriwaki, G. Pritschow, G. Ulsoy, and H. Van Brussel, "Reconfigurable manufacturing systems," CIRP Annals-Manufacturing Technology, vol. 48, pp. 527-540, 1999.

[28] T. Arai, Y. Aiyama, M. Sugi, and J. Ota, "Holonic assembly system with Plug and Produce," Computers in Industry, vol. 46, pp. $289-299,2001$.

[29] N. Okino, "Bionic manufacturing systems," presented at the Flexible manufacturing Systems: Past-Present-Future, 1993.

[30] K. Ueda, "A concept for bionic manufacturing systems based on DNA-type information," 1992, pp. 853-863.

[31] IDEAS-Deliverable1.4, "IDEAS MAS Framework," UNINOVA, Lisbon2011.

[32] IDEAS-Deliverable2.2, "IDEAS Module description language," Royal Institute of Technology, Stockholm2011.

[33] IDEAS-Deliverable2.1, "IDEAS Assembly Process Model," The University of Nottingham, Nottingham 2011. 